\title{
Bioavailability of generic $0.05 \%$ difluprednate emulsion in the aqueous humor, cornea, and conjunctiva of New Zealand rabbits after a single dose compared with commercial difluprednate
}

\author{
Arieh Mercado-Sesma ${ }^{1,2^{*}}$ (D), Angélica Contreras-Rubio ${ }^{1}$, Leopoldo Baiza-Durán ${ }^{1}$, Oscar Olvera-Montaño', \\ Mónica Miranda-Robles ${ }^{1}$ and José Bonilla-García'
}

\begin{abstract}
Background: To determine the concentration after a single dose of generic $0.05 \%$ difluprednate and commercial difluprednate in the aqueous humor, cornea, and conjunctiva of New Zealand rabbits, a preclinical study in 72 male New Zealand white rabbits was performed. A single dose (50 $\mu \mathrm{L})$ of two $0.05 \%$ difluprednate ophthalmic formulations was instilled in both eyes. Conjunctiva, cornea, and aqueous humor samples were collected at nine time points over $8 \mathrm{~h}$ (four animals per time point). The active metabolite of difluprednate, 17-difluoroprednisolonebutyrate (DFB), concentrations was quantified using HPLC.

Results: Measurable levels of DFB were quantified in all three ocular tissues. After a single instillation, the highest concentration of difluprednate was found between 30 and 60 min in the conjunctiva, cornea, and aqueous humor, respectively. There was no significant difference between both formulations in any tissue at any time point. After $3 \mathrm{~h}$, no metabolites of either emulsion were found in any tissue.

Conclusions: Difluprednate penetrates into different ocular tissues. Generic difluprednate has a similar pharmacokinetic profile compared with commercial difluprednate.
\end{abstract}

Keywords: Difluprednate, Pharmacokinetic, Difluprednate bioavailability, Ophthalmic corticosteroids

\section{Background}

Despite of the eye having immune privilege $[1,2]$ exist a lot of situations where an inflammatory response can be ignited. If an uncontrolled inflammatory ocular response appears, it can lead a patient to present unpleasant symptoms and signs: pain, edema, discomfort, photophobia, hyperemia, synechiae, and others. But above all can be associated with some complications, corneal edema, posterior capsule opacification, or cystoid macular edema [3-5]. Fortunately, it is possible to limit this

\footnotetext{
* Correspondence: arieh.mercado@sophia.com.mx

${ }^{1}$ Preclinical Research Department, Laboratorios Sophia S.A de C.V., Zapopan, Jalisco, México

${ }^{2}$ Departament of Health-Disease Sciences as Individual Process, Centro

Universitario de Tonala. Universidad de Guadalajara, Tonalá, Jalisco, México
}

obnoxious experience by the employment of anti-inflammatory medications, and their prophylactic use is a standard practice. Among anti-inflammatory medications, corticosteroids are the cornerstone in the treatment of this pathophysiologic process. However, most corticosteroids have a low ocular bioavailability, due in part to the characteristics of the cornea, limiting their effect [6, 7].

Difluprednate is a prednisolone derivative with structural modifications (fluorination at the $\mathrm{C} 6$ and $\mathrm{C} 9$ positions and replace 17-hydroxyl group with butyric acid) that enhanced and augmented its potency and anti-inflammatory activity, respectively. Furthermore, the substitution of the 21-hydroxyl group with acetic acid increased the corneal affinity and absorption $[8,9]$. 
Despite to be approved in other countries since 2008, in Mexico and Latin America, difluprednate ophthalmic emulsion is not commercially available. Hence be important to develop a new therapeutic option for the treatment of inflammatory diseases.

The objective of the present study was to compare the ocular pharmacokinetics of generic difluprednate and its commercial presentation in an animal model.

\section{Methods}

\section{Study design}

The study was a double-blinded preclinical study. Both the ophthalmologist investigator and biostatistician were blinded to the study medication.

\section{Animal model}

Seventy-two male New Zealand white rabbits, weighing between 2-3 kg, obtained from a local farm were used in this study. Animals with ocular abnormalities were excluded from the study. All rabbits were housed individually under controlled conditions, which included temperature ranging between 16 and $26{ }^{\circ} \mathrm{C}$, humidity between 40 and $70 \%$, and cycles of light/dark of $12 / 12 \mathrm{~h}$. Water ad libitum was arranged. Food consisted of standard alfalfa pellets. They were randomly allocated to both groups, so as to get four animals per time point.

\section{Drug administration and sample collection}

Animals were randomized to two groups to receive difluprednate emulsion $0.05 \%$ manufactured by two different laboratories: PRO-145 (Laboratorios Sophia S.A. de C.V, Guadalajara, Jalisco, México) or Durezol ${ }^{\odot}$ (Alcon Laboratories, Inc., Forth Worth, Texas, USA). A single $50-\mu \mathrm{L}$ eye drop of each drug formulation was instilled into the superior bulbar conjunctiva of both eyes. Sampling for drug concentrations were performed at the following time points after drug administration: 15,30 , and $60 \mathrm{~min} ; 1.5,2,3,4,6$, and $8 \mathrm{~h}$ ( $n=4$ rabbits per time point). The tissues taken for pharmacokinetic analysis were bulbar conjunctiva, cornea, and aqueous humor. The samples were obtained after animals were euthanized by an overdose of pentobarbital $(100 \mathrm{mg} / \mathrm{kg})$. Immediately after death, the bulbar conjunctiva, cornea, and aqueous humor were dissected, stored $\left(-20{ }^{\circ} \mathrm{C}\right)$, and processed separately.

\section{Drug assay}

The samples were assayed using a validated high performance liquid chromatography method (HPLC;
Waters corporation, Milford, MA, USA) The analytical column was C18 Gemini (Phenomenex NS655712-5) with a $5-\mu \mathrm{m}$ particle size and $150 \times 4.6 \mathrm{~mm}$ internal diameter, using a mobile phase of phosphate buffer and acetonitrile. The wavelength of detection was $240 \mathrm{~nm}$. The lower limit of detection was $4 \mathrm{ng} / \mathrm{mL}$ in the aqueous humor and $23.7 \mathrm{ng} / \mathrm{mL}$ in the cornea/ conjunctiva.

\section{Statistical analysis}

The concentration values are showed in mean and SD. Pharmacokinetic parameters $\left(C_{\max }, T_{\max }\right.$, and $\left.\mathrm{T}_{1 / 2}\right)$ were calculated by non-compartmental analysis using WinNonlin version 2.1 (Pharsight Corporation, Mountain View, CA, USA). The difference between groups was analyzed by Mann-Whitney test using SPSS version 19 (IBM Corporation., Armonk, NY, USA). A value $p<0.05$ was considered to be statistically significant.

\section{Results}

Concentrations of 17-difluoroprednisolone-butyrate (DFB) were detected in all tissues. The highest concentration was observed in the conjunctiva at $0.5-1 \mathrm{~h}$ in both formulations, and the lowest was detected after $4 \mathrm{~h}$. As expected, the concentration of DFB was different in the aqueous humor, cornea, and conjunctiva. There were no differences between the determinations of both formulations in the cornea and aqueous humor at any time point. There were statistical differences in DFB concentration measured in the conjunctiva at times $15 \mathrm{~min}$ and 2 and $4 \mathrm{~h}$; however, after $6 \mathrm{~h}$, both formulations were undetectable Fig. 1.

The pharmacokinetic parameters were similar in both formulations. The $\mathrm{T}_{\max }$ was found at $0.5-1 \mathrm{~h}$ in all tissues. There was no significant difference between formulations in terms for any parameter Table 1.

\section{Discussion}

Corticosteroids are a widely used topical ocular therapy. They are an important component of the treatment of diseases in which inflammatory response plays a central role, suppressing cellular infiltration and increasing the synthesis of lipocortins, mainly [6]. Difluprednate, a corticosteroid, mediates its antiinflammatory effects through the glucocorticoid receptor after intracellular distribution [10]. However, several factors are involved in ophthalmic drug efficacy, diffusion and distribution to targeted ocular tissues are of the most important [11, 12]. Due to its chemical structure, difluprednate ophthalmic emulsion has a high lipophilicity, which facilitates passage through the membranes, allowing a significant distribution to the 


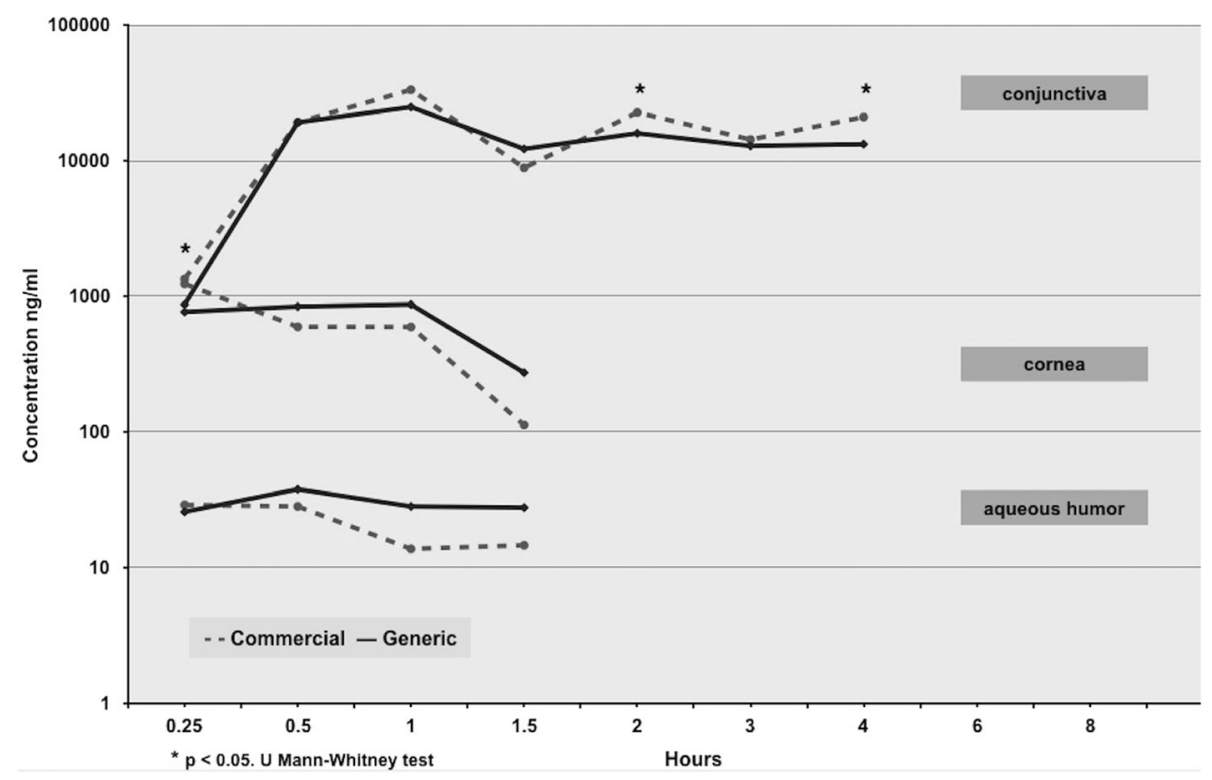

Fig. 1 Bioavailability of $0.05 \%$ generic vs commercial difluprednate in different ocular tissues

anterior and posterior segments, unlike early generations of corticosteroids [13], and clinical efficacy in medical settings $[14,15]$.

Ocular pharmacokinetics after a single instillation of difluprednate has been studied in rabbits. $T_{\max }$ in both studies was similar in aqueous humor, in spite of having used different methods of quantification $[13,16]$. In the present data, $\mathrm{T}_{\max }$ was similar in aqueous humor according to mentioned below.

The half-life $\left(\mathrm{T}_{1 / 2}\right)$ reported in the aqueous humor, conjunctiva, and cornea [13] was similar to the present results.

Notwithstanding that $\mathrm{C}_{\max }$ determined in the aqueous humor and cornea was similar that described in the previously mentioned, this parameter differed in conjunctiva's quantification. In the present study, the values were higher than those reported previously.

These discrepancies could be due to the methods used. Tajika et al. [13] quantified DFB concentration by HPLC using a radio labeled with tritium in contrast with the present data.
However, in the present study, the results were not dissimilar according to the comparator. Therefore, distribution of both formulations had the equivalent pharmacokinetic profile. Although the cornea is a major barrier, both formulations showed similar concentrations in all tissues at the present study. There are no other reports with similar methods evaluating the concentration of difluprednate in the conjunctiva.

The present data confirm that pigment does not affect the bioavailability of difluprednate. Difluprednate is distributed to the anterior and posterior segments via both the transcorneal and non-corneal absorption route [13, 16]. However, the distribution to anterior-posterior retina/choroid was not evaluated, and this is a weakness of the present study.

Although there was no significant difference in the pharmacokinetic profile between formulations used, the pharmacodynamic characteristics of generic difluprednate have not been determined yet. More studies are required to determine the security and efficacy of difluprednate in Mexican population.

Table 1 Pharmacokinetic parameters of difluprednate in the aqueous humor, cornea and conjunctiva after a single instillation

\begin{tabular}{|c|c|c|c|c|c|c|}
\hline \multirow{2}{*}{$\begin{array}{l}\text { PK } \\
\text { parameter }\end{array}$} & \multicolumn{2}{|c|}{ Aqueous humor } & \multicolumn{2}{|l|}{ Cornea } & \multicolumn{2}{|c|}{ Conjunctiva } \\
\hline & Generic & Commercial & Generic & Commercial & Generic & $\overline{C o m m e r c i a l}$ \\
\hline $\mathrm{T}_{\max }$ & 30 & 30 & 15 & 15 & 60 & 60 \\
\hline$C_{\max }$ & 35 & 27 & 1072 & 1255 & 25,288 & 31,351 \\
\hline $\mathrm{T}_{1 / 2}$ & 2.1 & 2.1 & 1.1 & 1.4 & 4.6 & 4.2 \\
\hline
\end{tabular}

Concentration in nanograms per milliliter

$T_{\max }$ time of maximum concentration, $C_{\max }$ maximum concentration, $T_{1 / 2}$ half-life 


\section{Conclusions}

Generic difluprednate has a similar pharmacokinetic profile compared with commercial difluprednate. Pigment does not affect the bioavailability of difluprednate which is distributed to the anterior and posterior segments via both the transcorneal and non-corneal absorption route.

\section{Abbreviations}

ARVO: Association for Research in Vision and Ophthalmology:

CICUALLS: Internal Committee for the Care and Use of Laboratory Animals of Sophia Laboratories; $C_{\text {max }}$ : Maximum concentration; DFB: 17-

Difluoroprednisolone-butyrate; GLP: Good Laboratory Practice; HPLC: High performance liquid chromatography; $T_{1 / 2}$ : Half-life; $T_{\max }$ : Time of maximum concentration

\section{Acknowledgements}

The authors would like to thank Alejandra Ríos M.D. for the medical writing and editorial assistance.

\section{Funding}

This trial was funded by Laboratorios Sophia S.A de C.V.

\section{Availability of data and materials}

Data will not be shared for protection.

\section{Authors' contributions}

MSA, OMO, ACR, and BDL participated in the design and writing of the study and data interpretation. MMR and BGJ participated in the data collection. MSA performed the statistical analysis of the data. All authors helped to draft the manuscript, and all authors read and approved the final manuscript.

\section{Competing interests}

All authors are employees of Laboratorios Sophia S.A de C.V. and declare that they have no competing interest.

\section{Consent for publication}

Not applicable.

\section{Ethics approval}

The protocol was performed in the animal facility belonging to Sophia Laboratories. It was reviewed and approved by the Internal Committee for the Care and Use of Laboratory Animals of Sophia Laboratories (CICUALLS) according to the guidelines for animal experimentation described by the Association for Research in Vision and Ophthalmology (ARVO) and Good Laboratory Practice (GLP).

\section{Publisher's Note}

Springer Nature remains neutral with regard to jurisdictional claims in published maps and institutional affiliations.

Received: 28 June 2016 Accepted: 10 March 2017

Published online: 21 March 2017

\section{References}

1. Streilein JW (2003) Ocular immune privilege: therapeutic opportunities from an experiment of nature. Nat Rev Immunol 3:879-889

2. Mochizuki M (2010) Regional immunity of the eye. Acta Ophthalmol 88:292-299

3. El-Harazi SM, Feldman RM (2001) Control of intraocular inflammation associated with cataract surgery. Opin Ophthalmol 12:4-8

4. Dennis EA, Norris PC (2015) Eicosanoid storm in infection and inflammation. Nat Rev 15:511-523

5. Huang JJ, Gaudio PA (eds) (2010) Ocular inflammatory disease and uveitis manual. Diagnosis and treatment. Wolters Kluwer. Lippincott Williams \& Wilkins, Baltimore

6. Rhen T, Cidlowski JA (2005) Antiinflammatory action of glucocorticoids-new mechanisms for old drugs. N Engl J Med 353:1711-23
7. McGee CN, Dean S, Danesh-Meyer H (2002) Locally administered ocular corticosteroids: benefits and risks. Drug Saf 25(1):33-55

8. Mulki L, Foster CS (2011) Difluprednate for inflammatory eye disorders. Drugs of today 47(5):327-333

9. Mithal C, Singh S, Gupta S, Mithal S (2012) Difluprednate: an overview. Del J Ophthalmol 23(3):165-168

10. Tajika T, Takahashi H, Sakai Y, Fujii H et al (2010) Metabolic profiles of difluprednate in rabbit ocular tissues after instillation of difluprednate ophthalmic emulsion. Xenobiotica 40(8):569-577

11. Worakul N, Robinson JR (1997) Ocular pharmacokinetics/ pharmacodynamics. Eur J Pharm Biopharma 44(1637):71-83

12. Novack GD, Robin AL (2016) Ocular pharmacology. J Clin Pharm 56(5):517-527

13. Tajika T, Isowaki A, Sakaki H (2011) Ocular distribution of difluprednate ophthalmic emulsion 0.05\% in rabbits. J Ocul Pharmacol Ther 27(1):43-49

14. Jamal KN, Callanan DG (2009) The role of difluprednate ophthalmic emulsion in clinical practice. Clin Opthalmol 3:381-390

15. Donnenfeld ED (2011) Difluprednate for the prevention of ocular inflammation postsurgery: an update. Clin Ophthalmol 5:811-816

16. Tajika T, Waki M, Tsuzuki M, Kida T et al (2011) Pharmacokinetic features of difluprednate ophthalmic emulsion in rabbits as determined by glucocorticoid receptor-binding bioassay. J Ocul Pharmacol Ther 27(1):29-34

\section{Submit your manuscript to a SpringerOpen ${ }^{\circ}$ journal and benefit from:}

- Convenient online submission

- Rigorous peer review

- Immediate publication on acceptance

- Open access: articles freely available online

- High visibility within the field

- Retaining the copyright to your article 